\title{
¿Existen Límites en la Decisión de los Padres Sobre el Tratamiento de sus Hijos?
}

\author{
MARÍA ELENA MCNAB C. ${ }^{1}$, JUAN PABLO BECA I. ${ }^{2}$ \\ 1. Estudiante de Medicina, Facultad de Medicina Clínica Alemana Universidad del Desarrollo. \\ 2. Centro de Bioética, Facultad de Medicina Clínica Alemana Universidad del Desarrollo.
}

\begin{abstract}
Are There Limits on Parental Decision-Making Over Children's Treatment?

In medical practice, there are situations where medical opinion differs from the patient's perception of their own benefit. This situation becomes more complex when the parents of a minor child are who deny their children life-saving treatment. It is necessary to determine how far professionals will fight over the management of a child's care if parents refuse treatment. To help in the development of criteria, three recently publicized cases are reviewed, including an analysis of the possible limits of parents to decide the treatment of their children. It is concluded that while it is vital to understand and respect the parents' beliefs, the fundamental duty is to the patient and their right to live. Therefore, it is justified to access the Courts when persuasion fails.

(Key words: Bioethics, Treatment refusal, parents, minors).

Rev Chil Pediatr 2010; 81 (6): 536-540

\section{RESUMEN}

En la práctica de la medicina nos encontramos ocasionalmente con situaciones en las que la opinión médica discrepa con la del paciente acerca de su mayor beneficio. Esta situación se torna aún más compleja cuando son los padres del paciente menor de edad quienes se niegan a que sus hijos reciban un tratamiento que le puede salvar la vida. Se hace necesario precisar hasta dónde luchar por la salud de un niño si sus padres rechazan los tratamientos. Con el propósito de contribuir a establecer criterios en este tema, se presentan tres casos públicos recientes y se analizan los posibles límites de la autoridad de los padres para decidir los tratamientos de sus hijos. Se concluye que es de vital importancia comprender y respetar las creencias de los padres del menor, pero que en último término nuestro compromiso fundamental es con el paciente y su derecho a la vida, razón por la cual se justifica, ante el fracaso de la persuasión, recurrir a los tribunales como última instancia.

(Palabras clave: Bioética, rechazo de tratamientos, padres, menores).

Rev Chil Pediatr 2010; 81 (6): 536-540
\end{abstract}

Trabajo recibido el 06 de mayo de 2010, devuelto para corregir el 29 de julio de 2010, segunda versión el 31 de agosto de 2010, aceptado para publicación el 02 de noviembre de 2010.

Correspondencia a:

Dr. Juan Pablo Beca I.

E-mail: jpbeca@udd.cl 
En la práctica médica hay situaciones en las cuales la voluntad de los pacientes o de sus responsables puede entrar en conflicto con las indicaciones médicas. Esta situación, siempre compleja, se agudiza cuando se trata de menores de edad, a quienes legalmente representan sus padres o tutores. Surge así el difícil problema de precisar hasta dónde debemos luchar por la salud del paciente menor si sus padres se oponen a la indicación de un tratamiento considerado médicamente indicado.

La participación de los niños en decisiones de salud, particularmente en investigación, debe ser respetada y estimulada, razón por la cual hoy se solicita el consentimiento informado de los padres junto al asentimiento o disentimiento del niño ${ }^{1}$. Sin embargo, ante decisiones de tratamiento, particularmente si son tratamientos necesarios para salvar su vida, se genera un conflicto de especial dificultad. En estas situaciones tanto los padres como los jueces pueden sobrepasar legítimamente la voluntad del menor y ordenar su tratamiento ${ }^{2}$. La razón de este criterio es que se considera que el bien del niño y su vida están por sobre otros motivos o razones. El comienzo público de estos conflictos se origina en 1982 con el caso Baby Doe, quien padecía de Síndrome de Down con atresia esofágica y fístula traqueo-esofágica y sus padres rechazaron la cirugía, por lo que falleció pocos días después $^{3}$. Este hecho abrió un amplio debate médico y judicial en Estados Unidos después del cual el Departamento de Salud y Servicios Humanos dictó la siguiente orden: "Dejar de alimentar y cuidar a niños discapacitados en forma discriminatoria está prohibido por ley federal", disposición a ponerse en práctica en todos los hospitales de Estados Unidos ${ }^{4}$. El caso, conocido como "Baby Doe Regulations", es uno de los grandes referentes en el tema ${ }^{5}$.

Recientemente en nuestro medio se hizo público el caso Robinson Gómez y coincidentemente se publicaron casos similares en la prensa norteamericana. Estos casos generan la necesidad de una reflexión en torno a los posibles límites de la autoridad de los padres para rechazar tratamiento. El objetivo de este artículo es analizar estos casos públicos desde un punto de vista bioético para contribuir a establecer criterios sobre este tema.

\section{Tres casos públicos recientes}

\section{Caso 1}

En el sur de Chile, un niño de 11 años fue tratado dos años antes con quimioterapia por una Leucemia Linfoblástica Aguda con buen resultado inicial. Presentó una recaída extramedular tardía con compromiso sólo testicular, con una probabilidad de curación de más de $50 \%$ si se trataba con quimioterapia. Sin embargo, el niño, en base a su mala experiencia previa con efectos adversos del tratamiento, se negó a ser tratado nuevamente con quimioterapia, decisión que fue apoyada por su madre quien rechazó el tratamiento y acudió a medicinas alternativas. Ante esta situación el médico tratante presentó un recurso de protección que fue inicialmente acogido por el juez del caso, dictamen que fue apelado a la Corte, tribunal que lo revocó estableciendo que "la situación se enmarca dentro de aquellos casos en que el Estado no puede -ni moral ni jurídicamente-, imponer un tratamiento médico que tiene altos costos humanos y poca garantía de efectividad". El niño falleció 7 meses después.

\section{Caso 2}

En 2009 Daniel Hauser, de 13 años, residente del estado de Minnesota sufre de un linfoma de Hodgkin. El menor declaró, después del primer curso de quimioterapia, no creer que su enfermedad fuese de gravedad y su familia lo instó a tratarse con medicina alternativa, basados en sus creencias religiosas de Nemenhah Band de los indios nativos de Norteamérica. El juez del distrito falló en su contra, dadas las evidencias médicas que indican que el tratamiento de quimioterapia y radioterapia, tiene en este caso un $90 \%$ de posibilidades de sobrevida. Se ordenó que el niño recibiese, bajo protección del Estado, los 6 ciclos de quimioterapia y las 12 sesiones de radioterapia indicadas. El abogado de la familia defendió su posición afirmando que la decisión del juez vulneraba la autoridad de los padres para decidir por sus hijos. Finalmente se completó el tratamiento y su control, 6 meses después, lo declaró recuperado y sin evidencias de linfoma. 


\section{Caso 3}

En el estado de Wisconsin, la madre de Kara de 11 años se negó por razones religiosas a acudir a un centro hospitalario ante la evidente gravedad de su hija, a pesar de más de una semana de presiones de otros familiares para hacerlo. Los padres pertenecían a un grupo religioso informal que rechaza la medicina convencional poniendo su esperanza sólo en la oración. La niña falleció por cetoacidosis diabética. Los padres fueron acusados por el Estado de homicidio de segundo grado, con lo cual pueden recibir condena de hasta 25 años de cárcel.

\section{Algunos conceptos para el análisis}

La autoridad de los padres en nuestra cultura occidental está basada en 3 presunciones: 1) los menores son todavía muy inmaduros para tomar decisiones importantes; 2) los padres siempre actúan buscando el mejor interés para el niño, dados sus lazos naturales de afecto, y 3) la autoridad de los padres es esencial para mantener la integridad de la familia ${ }^{6}$. Sin embargo, el segundo supuesto es refutable por la dificultad para definir el mejor interés del niño, de tal manera que es posible que los padres no siempre tomen las decisiones más correctas para sus hijos.

En la sociedad plural contemporánea la autonomía del paciente es ampliamente respetada, quedando atrás el modelo paternalista de la medicina, y la opción religiosa se acepta como un fundamento de las decisiones de las personas. Sin embargo, esto muchas veces dificulta las decisiones clínicas cuando se rechazan tratamientos o exámenes por seguir una determinada creencia religiosa o una interpretación que puede ser errónea. Cuando un médico se ve enfrentado a decisiones de limitación de tratamientos por una opción religiosa, su función es ser claro con el paciente sobre las posibles consecuencias de su decisión y ofrecerle otras alternativas terapéuticas. También puede buscar contacto con su autoridad religiosa para verificar si el paciente está haciendo una adecuada interpretación de su doctrina ${ }^{8}$. Sin embargo, estos criterios son sólo aplicables a pacientes adultos, porque frente a un menor de edad la situación cambia radicalmente. En este último caso es posible recurrir a autoridades judiciales, las que tienen atribuciones para autorizar o rechazar un procedimiento o tratamiento que se considere necesario para el niño, dado que carece de plena capacidad para tomar este tipo de decisiones.

Actualmente el rechazo a un determinado tratamiento médico se considera un derecho de los pacientes en base al principio de autonomía $^{9,10}$. Incluso esta alternativa está incluida en el proyecto de ley sobre derechos del paciente, aún en trámite legislativo en el Congreso de nuestro país ${ }^{11}$. Sin embargo, esta decisión supone plena capacidad para la comprensión del diagnóstico, su tratamiento, complicaciones y pronóstico. Si bien la mayoría de los adultos tiene desarrollada esta facultad de discernimiento, no la tienen los niños menores y es incompleta en adolescentes, particularmente durante la adolescencia temprana entre los 10 y 16 años. Por esta razón la decisión final de tratamiento es habitualmente delegada a los padres del menor ${ }^{12,13}$, pero esto supone que la decisión subrogada por los padres se orienta claramente al beneficio del hijo o hija.

Si bien las normas jurídicas de la mayoría de los países reconoce los 18 años como la edad en que los jóvenes poseen responsabilidad legal, la mayoría de los menores son concientes de sus actos y repercusiones desde mucho antes. Estudios psicológicos consideran a los menores de 12 años de edad como personas con pensamiento moral preconvencional, y entre los 12 y 15 años como individuos con mayor madurez moral en la mayoría de los adolescentes, aunque en algunos este proceso puede demorar más ${ }^{14}$. Sin embargo, niños con una enfermedad crónica, particularmente niños con cáncer como los dos primeros casos, maduran antes haciendo más discutibles los límites de edad. El problema es que en estas situaciones, a pesar de poseer mayor capacidad, también son muy influenciables. Es muy común que los jóvenes, especialmente en relación con tratamientos médicos, sigan el consejo de sus padres por miedo a discrepar de ellos en temas tan serios y vitales como son las enfermedades potencialmente mortales. Los jóvenes, a pesar de enfrentarse a sus progeni- 
tores ante muchas situaciones, cuando toman conciencia de la severidad de su diagnóstico, se tornan más vulnerables, dejando en la mayoría de los casos que decidan sus padres.

\section{Discusión}

La responsabilidad de los padres en relación a la salud de sus hijos es un tema que ha sido ampliamente analizado. Existe consenso en que los padres son los responsables del cuidado de sus hijos, lo cual incluye el cuidado de su salud y la toma de decisiones ante la enfermedad. Por esta razón, y en base a la alta probabilidad de curación de los casos analizados a modo de ejemplo, surge la necesidad de intentar responder la pregunta sobre los límites del poder de los padres para decidir sus tratamientos. Como criterio general se presume que los padres, además de asumir la crianza y educación de sus hijos, siempre buscan proteger su vida y no se oponen a tratamientos considerados médicamente necesarios para ellos. El problema es que su apreciación puede estar influida entre otros motivos por razones culturales, religiosas y miedo al sufrimiento, los que son válidos y respetables pero no definitorios de una decisión. El caso clásico y paradigmático de necesidad de limitar la autoridad paterna es el caso Baby Doe, con la doctrina jurídica correspondiente, que antepone el derecho a tratamiento del niño por sobre el poder de decisión de sus padres. Los padres deben ser respetados y su opinión debe ser considerada por cuanto ellos tienen un rol que va mucho más allá de su aporte biológico en la reproducción. Sin embargo, su autoridad no puede ir en desmedro de la integridad física o mental de sus hijos como ocurre en los casos de maltrato infantil o como puede ocurrir en decisiones que impiden tratamientos necesarios para salvar su vida. Los padres, como todos, pueden cometer errores y por eso su poder no puede ser considerado como absoluto.

La autonomía del adolescente es parcial y en desarrollo, dependiendo su nivel de muchos factores entre los cuales la experiencia de la enfermedad influye de manera relevante. $\mathrm{Si}$ bien hoy se acepta el concepto de menor maduro con sus derechos ${ }^{14}$, existen grados de madu- rez que no se pueden establecer con claridad ni sólo en base a la edad. Ante cada caso será necesario evaluar su capacidad de decisión. Por otra parte, el grado de capacidad necesario para rechazar tratamientos médicamente indicados por su eficacia es mayor al exigible para rechazar tratamientos menos necesarios o menos eficaces. En base a este criterio, ante terapias con buen pronóstico se acepta ignorar el rechazo del menor e imponer los tratamientos indicados, buscando siempre el mejor equilibrio entre la decisión del menor, la opinión de los padres y el mayor beneficio para el niño ${ }^{15,16}$. Se ha planteado respetar la autonomía del adolescente pero condicionado a que tome la "opción correcta", lo cual es discutible desde una mirada fundamentalmente autonomista ${ }^{15}$. En todo caso, el rechazo o disentimiento de un menor sólo sería aceptable para tratamientos menores y para su participación en investigación.

Lo ideal es buscar siempre una decisión compartida entre médicos y padres, con la aceptación o asentimiento del menor dependiendo de su edad. En muchos casos, como en los analizados en este artículo, por diversos factores fracasa la comunicación y la relación clínica con lo cual se hace imposible una decisión compartida. Si después de intentarlo no se llega a solucionar el problema se puede recurrir a un tercero, como es el recurso judicial, para que su resolución tome la decisión correspondiente ${ }^{17}$. Aunque estas situaciones son muy infrecuentes, es importante aceptar que no siempre se aceptan los criterios médicos, como ocurrió en el caso chileno, con su lamentable resultado.

En las tres situaciones analizadas, los padres rechazaron el tratamiento propuesto para sus hijos en base a experiencias negativas previas o a creencias religiosas. Esta última situación ha sido analizada extensamente, especialmente ante el rechazo a transfusiones sanguíneas en hijos de padres que profesan la religión Testigos de Jehová ${ }^{18}$. El criterio recomendado, entre otras instituciones por la Academia Americana de Pediatría (AAP), es comprender y respetar las creencias de los padres, pero considerar que por sobre ellas los niños tienen derecho a ser protegidos en su salud y a recibir tratamientos efectivos para la enfermedad ${ }^{19}$. En el caso de 
las experiencias negativas previas, como ocurrió en el primer caso presentado, es importante comprender lo que significó para el niño y su familia el tratamiento previo con severos efectos secundarios. Sin embargo, el deber médico es ofrecer la oportunidad de un tratamiento con probabilidades reales de lograr la curación de la enfermedad. Al respecto la AAP señala que los padres que al rechazar tratamientos médicos permiten la muerte de sus hijos, pueden ser demandados legalmente como ocurrió en el caso de Wisconsin. En el caso chileno de rechazo materno a la quimioterapia los tribunales asumieron una posición menos estricta afirmando que el estado no puede imponer un tratamiento médico que tiene altos costos humanos y poca garantía de efectividad. Esta aseveración resulta muy discutible si se considera que los especialistas aseguraron que la probabilidad de sobrevida era superior al $50 \%$ si el niño recibía el tratamiento propuesto.

A modo de conclusión, se considera que la autoridad de los padres para rechazar tratamientos para sus hijos está limitada cuando existe urgencia o riesgo vital. Si bien se debe intentar llegar a decisiones compartidas entre los padres y el médico tratante, con la aceptación del niño según su edad, las razones y motivaciones paternas deben entenderse como condicionadas al derecho de sus hijos a recibir tratamientos efectivos. Lo anterior se enmarca dentro del derecho fundamental de todo ser humano que es el derecho a la vida. De esta manera se responde a la obligación de actuar siempre buscando el mejor interés del menor y no sólo el de sus padres, aunque comprendiendo respetuosamente sus creencias, temores o experiencias previas.

\section{Agradecimientos}

Los autores agradecen a la Dra. Carmen Astete A. por su colaboración y acuciosa revisión del manuscrito.

\section{Referencias}

1.- Committee on Bioethics, American Academy of Pediatrics: Informed consent, parental permission, and assent in pediatric practice. Pediatrics 1995; 95: 314-7.
2.- Ross LF: Against the tide: Arguments against respecting a minor's refusal of efficacious life-saving treatment. Camb Q Healthc Ethics 2009; 18: 302-15.

3.- Caso Baby Doe, 2007. Disponible en http://bioetica. udesarrollo.cl/html/documentos/documentos/casobabydoe.pdf

4.- Lang GC: "Baby Doe"- a Medical Ethical issue. West J Med 1985; 142: 837-41.

5.- Pence GE: The Baby Doe Rules. Classic Cases in Medical Ethics. McGraw Hill, Birmingham: 2004; 220-222.

6.- Fallo Corte Apelaciones de Valdivia, 14 de Mayo 2009, causa Rol N 103-2009 FAM.

7.- Hui E: Parental refusal on life saving treatments for adolescents: Chinese familism in medical decision -making re-visited. Bioethics 2008; 22: 286-95.

8.- English DC: Addressing a Patient's Refusal of Care Based on Religious Beliefs. Am Fam Phycisian 2007; 76: 1393-4.

9.- Simon Lorda P. El consentimiento informado. Ed. Triacastela, Madrid, España 2000.

10.- Problemas prácticos del consentimiento informado. Cuadernos de la Fundación Victor Grifols i Lucas. Barcelona, España, 2002.

11.- Proyecto de Ley que regula los derechos y deberes que las personas tienen en relación con acciones vinculadas a su atención en salud. Mensaje $\mathrm{N}^{\circ}$ 223-354, 26 Julio 2006.

12.- Hickey, Kathryn BA, RN: Minors' Rights in Medical Decision Making. JONA's Healthcare Law, Ethics, and Regulation 2007; 9: 100-4.

13.- Buchanan AE, Brock DW: Deciding for Others. The Ethics of Surrogate Decision Making. Cambridge University Press, Cambridge U.K., 1990.

14.- Gracia D, Jarabo Y, Espildora N M, Ríos J: Toma de decisiones en el paciente menor de edad. Med Clin. (Barc) 2001; 117: 179-90.

15.- Sawye SS: Respecting Adolescents' Autonomy (as Long as They Make the Right Choice). J Adolesc Health 2010; 47: 113-4.

16.- Talati ED, Lang $C W$, Ross $L F$ : Reactions of Pediatricians to Refusals of Medical Treatment for Minors. J Adolesc Health 2010; 47: 126-32.

17.- Micah Hester D: Adolescent decisionmaking, part 2. Camb Q Healthc Ethics 2009; 18: 432.

18.- Layon A, D'Amico $R$, Caton D, Mollet $C$ : And the patient chose: Medical ethics and the case of Jehovah's Witness. Anesthesiol 1990; 73: 1258-62.

19.- Policy statement-Religious Objections to Medical Treatment. Policy Statement 1977, reafirmed 2009. Pediatrics 2009; 124: 845. 